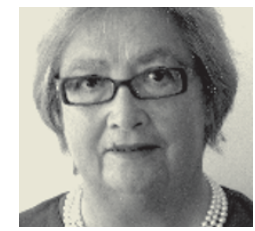

\title{
Vaccinate boys too
}

\section{HPV-associated cancers in men are on the rise. By not vaccinating boys we are failing to gain maximum health benefit, argues Margaret Stanley.}

$\mathrm{I}$ Infection with human papillomavirus (HPV) is the cause of almost all cervical cancers, as well as a significant number of cancers of the vulva and vagina. These links make us think of HPV as a women's health problem. But that's not the case.

HPV is also the main cause of cancers of the anus, tonsils and tongue, and is a significant contributor to cancers of the penis, larynx, head and neck. It is estimated to be the causal agent in $5 \%$ of all human cancers ${ }^{1}$. HPV is also the cause of genital warts, which are the commonest sexually transmitted viral disease with a lifetime risk of acquisition at $10 \%$ (ref. 2). Despite the virus's health impact on both sexes, most countries' HPV immunization programmes are exclusively for females. Only the United States, Canada and Australia recommend vaccination for boys and men as well. Indeed, Australia has recently announced that 12- and 13-yearold boys will be vaccinated starting in 2013 .

The rationale for these policies is straightforward. If a reduction in female cancer is the only public-health target, it is clear from mathematical models that male vaccination provides only a small added benefit. However, this approach fails to serve men who develop HPV-attributable cancers of the anus, penis, oral cavity and oropharynx, and who have an equivalent burden to women in terms of genital warts ${ }^{2}$.

\section{BEYOND THE CERVIX}

Anal carcinoma is a rare cancer, but its incidence among men aged 20-49 is on the rise. Rates of anal cancer are highest in men who have sex with men (MSM); the incidence in this group is estimated to be equivalent to that of cervical cancer in an unscreened population, and is even higher in HIV-infected MSM ${ }^{3}$.

Rates of HPV-associated cancers of the head and neck, known as oropharyngeal squamous cell carcinomas (OSCCs), have increased dramatically in both men and women in developed countries over the past 20-30 years. In the United States, incidence of HPV-related OSCC is higher in men than women, as is the prevalence of oral HPV infection. Unless preventive measures are put in place, the annual number of HPVpositive OSCC cases in the United States is predicted to exceed that of cervical cancer by 2020 (ref. 4).

The good news is that HPV vaccine trials have shown efficacy against HPV infection and related anogenital disease in men. It is less clear whether vaccination of males to prevent HPV infection is worth the expense. Most older economic models conclude that when female vaccination coverage is high, vaccination of boys is not cost effective ${ }^{5}$. Indeed, once female vaccination coverage exceeds $50 \%$, 'herd immunity' will eventually develop, and men who have sex only with women will be protected. Unfortunately, few countries have achieved the required rate of female HPV vaccination. Furthermore, MSM in this scenario receive little or no benefit from herd immunity and remain vulnerable to preventable HPV-associated disease. In the cost-effectiveness models, MSM represent too small a fraction of the population to justify general male vaccination. But not all national immunization programmes meet strict cost-effectiveness criteria. Vaccination against meningococcal infection in children is not cost effective, for example, but society accepts it because the prevention of such a serious disease is a worthwhile public-health goal.

\section{TARGETING MEN}

One way to make a male HPV vaccine programme cost effective would be to target MSM exclusively ${ }^{6}$. But such a limited vaccination policy would raise important issues of access, equality and ethics. It would also lengthen the time needed to establish herd immunity, and until that time, unvaccinated men will continue to develop preventable HPV-associated cancers. Anyone implementing a strategy targeting MSM would face the tricky question of what is the best age for such a vaccine. For optimal vaccine impact, MSM would need to be reached in early adolescence, before they start having sex. But questioning boys in this age group about their sexual orientation would produce highly unreliable results, in large part because orientation is not yet firmly established. (Such questioning would also be likely to elicit parental outrage.) But the alternative - targeting men in their late teens - would lower the vaccine's effectiveness because this age group includes large numbers of sexually active men who have had ample occasion for HPV exposure. Moreover, requiring the disclosure of sexual orientation as a prerequisite for immunization is ethically questionable.

In many developed countries, including those in western Europe, the burden of $\mathrm{HPV}$-associated cancers in men is now comparable to that in women ${ }^{2}$. Cervical cancer, the dominant HPV-related cancer in women, can be prevented through vaccination and screening, but there is no screening for anal cancer or OSCC - serious diseases that tend to occur in younger age groups than cervical cancer, present at a later stage with associated mortality, and show significant morbidity with impaired quality of life after therapy. All men, regardless of sexual orientation, face a significant and increasing risk of $\mathrm{HPV}$-associated disease. It is not ethical, fair or socially responsible to have a public-health policy that forces men to rely on herd immunity, which won't be reached for decades. Let's start vaccinating men now. -

Margaret Stanley is director of research in the Department of Pathology, University of Cambridge, UK.

email:mas1001@cam.ac.uk

1. de Martel, C. et al. Lancet Oncol. 13, 607-615 (2012).

2. Hartwig, S. et al. BMC Cancer 12, 30 (2012).

3. Frisch, M. Dan. Med. Bull. 49, 194-209 (2002).

4. Chaturvedi, A. K. et al. J. Clin. Oncol. 29, 4294-4301 (2011).

5. Garnett, G. P. J. Infect. Dis. 191, S97-S106 (2005).

6. Kim, J. J. N. Engl. J. Med. 364, 393-395 (2011). 